\title{
A STUDY OF THE IRRADIATION OF SECONDARIES OF ALGOL BINARIES
}

\author{
A. GIMENEZ AND A. CLARET \\ Instituto de Astrofísica de Andalucía \\ Apurtado 2144 \\ 18080 Granada \\ Spain
}

(Not reviewed)

In order to investigate the effects of irradiation on the spectra of the cooler components of Algol binaries, a modified version of the Upsalla Model Atmosphere code (U.M.A.) has been used. The upper boundary condition was changed to take into account the external radiation field and an automatic procedure to prevent numerical oscillations was introduced. In order to simulate the external flux from the hot component we have used the Kurucz grid interpolated for the wavelenghts adopted in U.M.A. code. Since secondary stars of Algoltype binaries normally have deep convective layers, we impose the condition that the entropy of these zones must be the same in the irradiated and in the non-irradiated models.

The bolometric albedo calculated from our models depends on the relative distance between the components, the relative fluxes and the incidence angle. There is also a correlation with $\log g$ and $\log [\mathbf{A} / \mathrm{H}]$. We have furthermore found that these albedos are different from unity and in good agreement with the observations.

As expected from previons works, irradiated spectra are very different from non-irradiated ones with the same effective temperature and $\log \mathrm{g}$. However, in some regions of the spectrum, they are much better reproduced by models with the same stellar characteristics but lower metallicity. This means that the use of non-irradiated atmosphere models in the study of secondaries of Algols inay introduce systematic errors in the interpretation of basic parameters, and in particular in the determination of metal content.

It can be noticed that if the incidence angle is small and/or the external flux is much greater than the original one, some absorption lines can be filled by emission. 'This can be related to irradiation itself or perhaps with the LTE assumption adopted in the atmosphere model.

Finally, limb-darkening is also changed by irradiation and depends, like the bolometric albedo, on the external flux, incidence angle and relative distance of the component stars. In particular we find that linear limb-darkening laws do not fit the atmospheric irradiated model. 